1 Department of Haematology, Oxford University Hospitals NHS Foundation Trust, Oxford, UK

2 Thrombosis \& Haemophilia Centre, Guy's \& St Thomas NHS Foundation Trust \& King's College London, UK

3 Critical Care Unit, Salford Royal NHS Foundation Trust, Stott Lane, Salford, UK

4 Division of Infection, Immunity and Respiratory Medicine, University of Manchester, UK

5 Department of Women and Children's Health, King's College London, UK

6 National Institute for Health and Care Excellence (NICE), London, UK

Correspondence to J Karpusheff Justine.Karpusheff@nice.org.uk

Cite this as: BMJ 2021;375:n2195

http://dx.doi.org/10.1136/bmj.n2195

Published: 01 October 2021

\title{
GUIDELINES
}

\section{Vaccine induced immune thrombocytopenia and thrombosis: summary of NICE guidance}

\section{Sue Pavord, ${ }^{1}$ Beverley J Hunt, ${ }^{2}$ Daniel Horner, ${ }^{3,4}$ Susan Bewley, ${ }^{5}$ Justine Karpusheff ${ }^{6}$, on behalf of the Guideline Committee}

\section{What you need to know}

- Vaccine induced immune thrombocytopenia and thrombosis (VITT) is caused by anti-platelet factor 4 (PF4) antibodies that arise following covid-19 vaccination and lead to intense activation of platelets and the coagulation system

- Symptoms begin 5 to 30 days after covid-19 vaccination and include severe or unusual headaches, new unexplained pinprick bruising or bleeding, shortness of breath, leg swelling, or persistent abdominal pain

- Thrombosis affects the cerebral veins in $50 \%$ of cases, but any arterial or venous vascular bed may be involved and around one third of patients have thrombosis in multiple sites

- Management includes anticoagulation with non-heparin based anticoagulants, which should be started as soon as possible, and intravenous immunoglobulin

- VITT is rare, but thrombotic sequelae can be life threatening and require unconventional management. Report all cases urgently and inform vaccine recipients of when to seek medical attention

The UK vaccination programme has prevented thousands of deaths from infection with SARS-CoV-2, but has been accompanied by new vaccine related complications. The most serious of these is a novel disorder, described as vaccine induced immune thrombocytopenia and thrombosis (VITT). This immune mediated condition is caused by development of pathological anti-platelet factor 4 (PF4) antibodies following vaccination against covid-19, which leads to intense activation of platelets and the coagulation system. The subsequent clinical syndrome often presents with life threatening thrombosis and secondary haemorrhage, which are challenging to manage in the context of new biochemical coagulopathy.

This article summarises the most recent recommendations from the rapid covid-19 guideline produced by the National Institute for Health and Care Excellence (NICE). ${ }^{1}$

\section{Recommendations}

NICE recommendations are based on systematic reviews of best available evidence. This guideline was developed using an interim process and methods for guidelines developed in response to health and social care emergencies. These included convening an independent advisory expert panel, scoping, identifying, and reviewing evidence, panel discussion of recommendations, targeted peer review with stakeholders, and conducting an equalities impact assessment. Available evidence was limited, given the novel nature of this condition, and recommendations were based mainly on expert opinion.

\section{Incidence and pathogenesis of VITT}

VITT is rare, with an incidence of 14.9 per million after first or unknown dose of covid-19 vaccine, and 1.8 cases per million after second doses. ${ }^{2}$ Some 416 major thromboembolic events with concurrent thrombocytopenia were recorded in the MHRA's weekly summary of yellow card reporting ${ }^{2}$ on 1 September 2021.

VITT can affect people of all ages, but summary data from UK yellow card reporting (1 September 2021) indicate that after a first dose, the incidence rate in adults aged 18-49 years is twice that of adults 50 years and older.

\section{Onset of symptoms after vaccination}

The guideline emphasises the importance of providing information to vaccine recipients to help them recognise common and unusual symptoms arising from covid-19 vaccination, and to know when and how to seek help (box 1). Patients should be encouraged to report any unusual symptoms that start five or more days after vaccination.

Box 1: Characteristic symptoms of VITT, starting 5-30 days after vaccination

- New severe headache, which gets worse and does not respond to simple painkillers

- Unusual headache that seems worse when lying down or bending over, or may be accompanied by blurred vision, nausea and vomiting, difficulty with speech, weakness, drowsiness, or seizures

- New unexplained pinprick bruising or bleeding

- Shortness of breath, chest pain, leg swelling, or persistent abdominal pain

- Pain at the injection site, fatigue, headache, and fever commonly occur immediately after the vaccine. These symptoms usually resolve within 2-3 days, and are not related to VITT. Rarely inflammatory cardiac and neurological complications have been reported after covid-19 vaccination; these are also unrelated to $\mathrm{VITT}^{2}$

\section{Investigations}

The guideline makes recommendations for healthcare professionals caring for people who have suspected VITT in any setting. The UK Expert Haematology 
Group has developed consensus diagnostic criteria. ${ }^{3}$ These were not included in the initial version of the NG20o guideline but have been included here for information (table 1).

\begin{tabular}{|c|c|}
\hline \multicolumn{2}{|c|}{$\begin{array}{l}\text { The UK Haematology Expert Group has developed consensus diagnostic criteria, suggesting that one or more of the following criteria missing or unfulfilled gives a probable, } \\
\text { possible, or unlikely diagnosis }\end{array}$} \\
\hline Probable & $\begin{array}{l}\text { - D dimer }>4000 \text { FEU but one criterion not fulfilled (timing, thrombosis, thrombocytopenia, } \\
\text { anti-PF4 Abs), or } \\
\text { - D dimer unknown or 2000-4000 FEU with all other criteria present }\end{array}$ \\
\hline Possible & $\begin{array}{l}\text { - D dimer unknown or 2000-4000 FEU with one other criterion not fulfilled, or } \\
\text { - Two other criteria not fulfilled (timing, thrombosis, thrombocytopenia, anti-PF4 Abs) }\end{array}$ \\
\hline Unlikely & $\begin{array}{l}\text { - Platelet count }<150 \times 109 / \mathrm{L} \text { without thrombosis with D dimer }<2000 \mathrm{FEU} \text {, or } \\
\text { - Thrombosis with platelet count }>150 \times 109 / \mathrm{L} \text { and D dimer }<2000 \mathrm{FEU} \text {, regardless of anti-PF4 } \\
\text { Ab result, and/or } \\
\text { - Alternative diagnosis more likely }\end{array}$ \\
\hline
\end{tabular}

- When assessing people with suspected VITT, ask about their vaccination history, take into account their overall clinical condition:

- Refer people who are acutely unwell to the emergency department immediately, or

- Perform initial tests (full blood count) in primary care if

$\square$ the person is not acutely unwell, and

$\square$ same day test results can be obtained, and

$\square$ if they show thrombocytopenia, the person can be referred to the emergency department immediately.

- If thrombocytopenia is present, refer the patient to secondary care immediately. VITT is a potentially life threatening condition and further rapid investigations are required to assess diagnostic probability and inform early management.

- If the full blood count confirms thrombocytopenia, or a strong clinical suspicion of VITT remains, undertake the following tests in secondary care:

- a coagulation screen, including Clauss fibrinogen assay and D-dimer measurement

- a blood film, to confirm true thrombocytopenia and identify potential alternative diagnoses.

- Some $5 \%$ of patients with VITT have a platelet count in the normal range at presentation but which subsequently falls below normal. If a high clinical suspicion of VITT remains, the platelet count should be repeated after two to three days.

- For people with a high clinical suspicion of VITT, perform same day imaging, based on location of symptoms, to confirm the site of thrombosis before starting treatment. Imaging may include head computed tomography (CT) venogram or magnetic resonance angiography to identify cerebral venous sinus thrombosis (CVST); abdominal ultrasound or venogram to identify portal or splanchnic vein thrombosis; CT pulmonary angiography to identify pulmonary embolism; and duplex compression ultrasonography to identify deep vein thrombosis in the lower or upper limbs.

- For all people with probable VITT, use an enzyme linked immunosorbent assay (ELISA) for $\mathrm{PF}_{4}$ antibodies to confirm diagnosis. If other diagnostic criteria are fulfilled, treatment should commence while waiting for the result. False negative anti-PF4 antibodies are unusual but may occur.

\section{Management}

- Explain to the person and their family members or carers (if appropriate) what a diagnosis of VITT means. Discuss treatments and possible outcomes.

- Involve a clinical haematologist when making decisions about starting and adding treatments.

The UK Expert Haematology Panel has produced a patient information leaflet about vaccine induced thrombosis and thrombocytopenia, ${ }^{4}$ which is available on Thrombosis UK's website (https://thrombosisuk.org/information-fact-sheets.php).

\section{Managing thrombosis and the immune response}

For people with suspected VITT, immediately give $1 \mathrm{~g} / \mathrm{kg}$ intravenous immunoglobulin to help remove the anti-PF4 antibodies, prevent platelet activation, and improve the platelet count. For people with VITT and confirmed thrombosis, therapeutic anticoagulation should be started as soon as possible after assessment of the bleeding risk and once the benefits outweigh the risks.

The guideline recommendation at the time of publication is to use non-heparin based anticoagulants in case of cross reactivity with the sister condition, heparin induced thrombocytopenia. If using argatroban, see guideline for additional considerations. ${ }^{1}$

For people with VITT without confirmed thrombosis, but who have thrombocytopenia with very high D-dimer and a positive ELISA for PF4 antibodies, consider venous thromboembolism (VTE) thromboprophylaxis. Give non-heparin drugs such as direct oral anticoagulants, fondaparinux, or danaparoid sodium, and regularly reassess the benefits and risks of thromboprophylaxis. 
- Consider fibrinogen replacement therapy with fibrinogen concentrate or cryoprecipitate to maintain a level of fibrinogen of at least $1.5 \mathrm{~g} / \mathrm{L}$.

\section{Additional considerations for thrombosis}

- Discuss the need for early transfer to specialist care, including for surgical interventions, with relevant specialists based on the location of the thrombosis. Take into account the person's clinical condition when determining the urgency of any transfer.

In particular, be aware that some people with cerebral venous sinus thrombosis caused by VITT experience rapid deterioration after appearing clinically well and may benefit from pre-emptive transfer to a centre with neuroscience services.

- For people with VITT who need surgery to treat the thrombosis and who are at higher risk of bleeding because of low platelets or low fibrinogen, give platelet transfusion or fibrinogen replacement.

- For people with VITT who have a very low platelet count (under $30 \times 10^{9} / \mathrm{L}$ ), consider one of the following alternative anticoagulation strategies that may reduce the risk of bleeding:
- a critical illness dose of argatroban, or

- a therapeutic dose of argatroban, plus platelet transfusion.

\section{Further treatment}

- Consider adding corticosteroids if intravenous immunoglobulin treatment is insufficient (that is, if there is progression of thrombosis or the platelet count does not rise to an acceptable level).

- Consider plasma exchange with fresh frozen plasma (one volume exchange a day) as an alternative to a second dose of intravenous immunoglobulin.

For people with VITT who have signs of poor prognosis, consider an intensive treatment strategy of plasma exchange and high dose steroids.

- Consider rituximab for people with VITT that has not responded to a second dose of intravenous immunoglobulin or plasma exchange.

The guideline gives advice on the follow-up of patients with confirmed VITT after discharge from hospital, as summarised in table 2.

\section{Table 2 | Follow-up and monitoring}

\section{All patients with confirmed VITT}

Patients should be kept under the care of the haematology department. Close monitoring is required after discharge

\begin{tabular}{ll}
\hline $1-2$ weeks & Check D-dimer and platelet count every 2 to 3 days \\
\hline 1-4 weeks & Perform anti-PF4 ELISA weekly \\
\hline 4 weeks-6 months & Perform anti-PF4 ELISA monthly \\
\hline 6 months onwards & Perform anti PF4-ELISA every 3 months \\
\hline Patients with confirmed venous thrombosis & \\
\hline At least 3 months after discharge and until anti-PF4 antibodies are no longer detected & Continue anticoagulation \\
\hline For patients with coronary artery thrombosis or arterial thrombosis in vessels without atherosclerosis \\
\hline At least 1 month & Continue anticoagulation and consider adding antiplatelet agents \\
\hline
\end{tabular}

\section{Reporting cases}

- Code and record all diagnoses and all probable, confirmed, and relapsed cases reported, using the MHRA's covid-19 Yellow Card scheme. ${ }^{5}$

- In addition, cases can be reported via the UK Expert Haematology Panel and Public Health England's clinical reporting scheme. ${ }^{6}$

\section{Guidelines into practice}

- Can you describe the presenting clinical, temporal, and biochemical features of VITT? How would you ensure appropriate early investigation in suspected cases, to provide a timely diagnosis and prompt treatment?

- VITT is a new condition with emerging evidence. How do you ensure contribution towards national datasets, to inform future guidance?

- VITT mainly affects people who are previously fit and healthy. They may have notable psychological challenges to face after diagnosis. How do you best support people and their families who have experienced VITT?

\section{Further information on the guidance}

The guideline is part of a series of rapid guidelines on coronavirus (covid-19), developed using interim methods

https://www.nice.org.uk/process/pmg2o/resources/appendix-l-interim process-and-methods-for-guidelines-developed-in-response-to-healthand-social-care-emergencies-8779776589/chapter/introduction-andoverview. ${ }^{7}$ The recommendations are based on evidence available when the guideline was published on 29 July 2021, it also draws on guidance developed by the UK Haematology Expert Group plus expert opinion. Recommendations will be reviewed and updated as the knowledge base and expert experience develops. Readers should refer to the full guideline on the NICE website for the latest version of the guidance (https://www.nice.org.uk/guidance/ng20o) and see the NICE coronavirus page (https://www.nice.org.uk/covid-19) for additional NICE rapid guidelines relating to covid-19.

\section{How patients were involved in the creation of this article}

Expert advisory panel members involved in this guideline included lay members who contributed to the formulation of the recommendations summarised here. One lay member of the panel Simon Race, reviewed the draft article and provided comments. 
Acknowledgments: We would like to acknowledge the members of the independent advisory expert panel, and staff at NICE for their invaluable contributions and for working at pace to produce this guideline.

Contributors: The members of the Guideline Committee were (shown alphabetically): Susan Bewley (chair), Marie-Christine Bielsky, Daniel Horner, Beverley Hunt, Will Lester, Michael Makris, Sue Pavord, Gary Peters, Simon Race, Stacey Ridpath, Marie Scully, Waqaar Shah, Astrid Ullich-Boereboom.

Funding: SP is an employee of Oxford University Hospitals NHS Foundation Trust.

Competing Interests: Declaration of interests based on NICE's policy on conflicts of interests is available at https://www.nice.org.uk/Media/Default/About/Who-we-are/Policies-and-procedures/code-of-practicefor-declaring-and-managing-conflicts-of-interest.pdf.

The guideline authors' full statements can be viewed at https://www.nice.org.uk/guidance/ng200/documents/expert-advisers

1 National Institute for Health and Care Excellence. COVID-19 rapid guideline: vaccine-induced immune thrombocytopenia and thrombosis (VITT). NICE guideline NG200. 2021.

https://www.nice.org.uk/guidance/ng200

2 Medicines \& Healthcare products Regulatory Agency. Coronavirus vaccine-weekly summary of Yellow Card reporting. 2021. https://www.gov.uk/government/publications/coronavirus-covid19-vaccine-adverse-reactions/coronavirus-vaccine-summary-of-yellow-card-reporting

3 Pavord S, Scully M, Hunt B, et al. Clinical features of vaccine-induced immune thrombocytopenia and thrombosis. N Engl J Med 2021. Doi:doi: 10.1056/NEJMoa2109908. https://www.nejm.org/doi/full/10.1056/NEJMoa2109908

4 UK Expert Haematology Panel. COVID-19 vaccine immune thrombosis and thrombocytopenia VITT. Information for patients. 2021 vitt_patient_information_v4-20210420.pdf (b-s-h.org.uk)

5 MHRA. Coronavirus Yellow Card reporting site. 2021. https://coronavirus-yellowcard.mhra.gov.uk/

6 Public Health England. Haematologic adverse events following Immunisation to covid-19. 2021. https://snapsurvey.phe.org.uk/snapwebhost/s.asp?k=161706705032

7 National Institute for Health and Care Excellence. Developing NICE guidelines: the manual. Appendix L: Interim process and methods for guidelines developed in response to health and social care emergencies. 2020. 